\title{
Phenomic and metabolomic responses of roots to cadmium reveal contrasting resistance strategies in two rice cultivars (Oryza sativa L.)
}

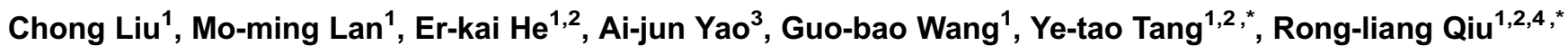 \\ 1 School of Environmental Science and Engineering, Guangdong Provincial Key Laboratory for Environmental Pollution Control and \\ Remediation Technology, Sun Yat-sen University, Guangzhou 510006, China \\ 2 Guangdong Provincial Engineering Research Center for Heavy Metal Contaminated Soil Remediation, Sun Yat-sen University, \\ Guangzhou 510006, China
}

3 School of Geography and Planning, Sun Yat-sen University, Guangzhou 510275, China

4 Guangdong Laboratory for Lingnan Modern Agriculture, College of Natural Resources and Environment, South China Agricultural University, Guangzhou 510642, China

\section{H I G H L I G H T S}

- We compared the phenomic and exudate metabolomic responses of roots of two rice cultivars to Cd.

- JY841 suffered serious root membrane damage and up-regulated phenylethanoid glycosides.

- TY816 up-regulated lipids and fatty acids to actively cope with oxidative stress.

- Reprogramming of root architecture and exudates contributed to contrasting $\mathrm{Cd}$ uptake.

\section{ARTICLE INFO}

Article history:

Received December 10, 2020

Revised March 15, 2021

Accepted March 20, 2021

Keywords:

Root morphology

Exudates

Cadmium

Rhizosphere

Metabolite
GRAPHICAL ABSTRACT

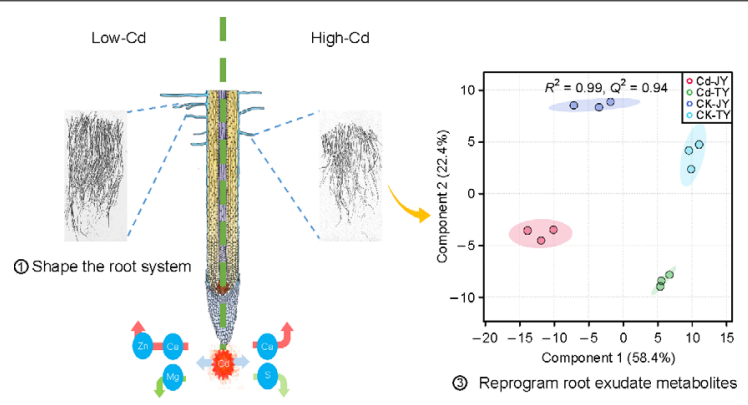

A B S T R A C T

To cope with heavy metal stress, plant root systems undergo root structure modification and release of multifarious metabolites. Elucidation of the resistance strategies to heavy metals mediated by the root system is crucial to comprehend the resistance mechanisms of plants. Here two rice cultivars with contrasting grain cadmium (Cd) accumulation traits were selected and the responses of their root systems to $\mathrm{Cd}$ stress were evaluated by morphological and metabolomics analysis. The phenomic and metabolomic responses of the root system varied between the two cultivars under $\mathrm{Cd}$ stress. The low-Cd accumulation rice cultivar (TY816) had a more highly developed root system that coped with Cd stress $(10 \mu \mathrm{M})$ by maintaining high root activity, while the root cells of the high-Cd accumulation cultivar (JY841) lost viability due to excessive Cd accumulation. TY816 upregulated lipids and fatty acids to reduce Cd uptake, whereas JY841 upregulated phenylethanoid glycosides to cope with Cd-induced oxidative stress. The combination of metabolomics and phenomics revealed that rice roots employ multiple strategies to increase their tolerance of Cd-induced oxidative stress. Differing capacities to shape the root system architecture and reprogram root exudate metabolites may contribute to the contrasting Cd accumulation abilities between JY841 and TY816.

(c) Higher Education Press 2021

\footnotetext{
* Corresponding authors

E-mail address: eestyt@mail.sysu.edu.cn (Y.T. Tang); eesqrl@mail.sysu.edu.cn (R.L. Qiu)
} 


\section{Introduction}

Heavy metal contamination in soil is of growing concern in China due to the potential risks to food security. According to a national soil contamination survey, $82 \%$ of China's farmland contains inorganic pollutants, among which cadmium (Cd) is the most prevalent (Zhao et al., 2015). Cd contamination in soil has resulted in $\mathrm{Cd}$ accumulation in cereal crops grown in China (Hu et al., 2016). Rice, which is a staple food for more than $50 \%$ of the world's population, has a tendency to accumulate $\mathrm{Cd}$. Subsequently, eating rice contaminated by $\mathrm{Cd}$ has become the main contribution to dietary $\mathrm{Cd}$ intake in rice-dependent populations, especially in tropical and subtropical countries that consume $\sim 90 \%$ of the rice grown in the world (Hu et al., 2016).

Due to the toxic potential of $\mathrm{Cd}$ to humans, the physiological processes of $\mathrm{Cd}$ behavior in the soil-rice plant system have been well studied. $\mathrm{Cd}$ is translocated from the soil to rice grains via several processes including root uptake, xylem loading, intervascular transfer, and remobilization via phloem (Ueno. et al., 2010; Li et al., 2017). This basic knowledge has led to further research on internal and external mechanisms for regulating $\mathrm{Cd}$ levels in rice grains, including reducing $\mathrm{Cd}$ bioavailability in soil, screening for low-accumulation cultivars, and manipulating transporter gene expression. As the first step for $\mathrm{Cd}$ entry into rice grain, root uptake is affected by variation in root morphology, which plays a key role in the $\mathrm{Cd}$ pool in the root system (Harris and Taylor, 2013). Huang et al. (2019) found that low-Cd rice cultivars have a lower number of root tips (NRTs) per root surface area (RSA). Positive correlations between root morphology and $\mathrm{Cd}$ accumulation have been reported for spring wheat and hot peppers (Huang et al., 2015; Liang et al., 2017). However, there is limited research focusing on the potential function of the root system in regulating $\mathrm{Cd}$ resistance ability in various rice cultivars. In addition, there is a lack of comprehensive evaluation of root morphology in response to $\mathrm{Cd}$.

The chemical behavior of $\mathrm{Cd}$ in the rhizosphere is the main factor influencing $\mathrm{Cd}$ uptake by plants. Root exudates contribute to the difference between the rhizosphere and bulk soil by altering soil biochemistry (Walker et al., 2003). As the first barrier to $\mathrm{Cd}$ uptake, plant root exudates are altered to adapt to Cd stress (Chen et al., 2017). Root exudates play crucial roles in the detoxification and accumulation of $\mathrm{Cd}$ in plants. However, the interaction between root exudates and Cd uptake by plants is still a matter of debate. For example, root exudates of oxalate and tartaric acid are thought to reduce phytotoxic $\mathrm{Cd}^{2+}$ and promote the transport of $\mathrm{Cd}$ in the hyperaccumulating ecotype Sedum alfredii (Tao et al., 2016; Tao et al., 2019). Nevertheless, low-Cd tomato cultivars may enhance $\mathrm{Cd}$ resistance and prevent Cd entry into roots by secreting oxalate (Zhu et al., 2011). Using two rice cultivars with different $\mathrm{Cd}$ accumulating abilities, $\mathrm{Fu}$ et al. (2018) observed more amino acids in the root exudates of high-Cdaccumulating rice than in low-Cd-accumulating rice. Thus, an assembly of various root exudate metabolites, which are plant and stress dependent, may contribute to $\mathrm{Cd}$ resistance in plants. Some studies have examined the relationships between specific root exudates and Cd uptake in rice (Lin et al., 2003; Liu et al., 2007). However, the role of various root exudate metabolites in $\mathrm{Cd}$ resistance and uptake in rice, as well as the underlying mechanism, remain less explored. Untargeted metabolomics allows profiling of the global metabolome, i.e., the identification and (semi-) quantification of thousands of metabolites in a plant. Metabolomics, being "downstream" from genomics and proteomics, has the closest link to phenotype (Zhang et al., 2019). Thus, it is potentially a powerful tool for revealing the molecular response of root exudate-mediated $\mathrm{Cd}$ resistance in rice.

This work aimed to reveal the phenomic and metabolomic responses of the rice root system under $\mathrm{Cd}$ stress to gain a deeper understanding of the $\mathrm{Cd}$-induced response in the rhizosphere from a "downstream" level. In this study, two rice varieties with distinct $\mathrm{Cd}$ accumulation capacities were selected and the effects of $\mathrm{Cd}$ stress on their root architecture and root exudates were investigated. Root phenomics and nontargeted UPLC-ESI-Q-TOF-MS-based root exudate metabolomics were employed to evaluate and compare the physiological responses induced by $\mathrm{Cd}$ in the root system between the two rice cultivars. Our findings could provide new insight for understanding the resistance mechanisms and abilities of different rice cultivars to tolerate $\mathrm{Cd}$.

\section{Material and methods}

\subsection{Plant culture and growth conditions}

Two rice cultivars with contrasting grain $\mathrm{Cd}$ accumulation traits, namely Jianyou841 (Xian/Indica, JY841) and Teyou816 (Xian/Indica, TY816), were selected for this study. In a previous study, JY841 was identified as a stable low-Cd cultivar while TY816 was identified as a high-Cd cultivar among 51 rice cultivars by a field plot test performed over two crop seasons (Chi et al., 2018). These two cultivars were used to explore the mechanisms of cultivar-specific differences in $\mathrm{Cd}$ accumulation and root systems.

Rice seeds were sterilized with $30 \% \mathrm{H}_{2} \mathrm{O}_{2}$ for 30 min and then $0.5 \%$ sodium hypochlorite solution for $24 \mathrm{~h}$. Seeds were then soaked in Milli-Q water and placed in darkness at $26^{\circ} \mathrm{C}$ for $24 \mathrm{~h}$ to germinate. One-week-old seedlings of a homogeneous size were transplanted to $2.0 \mathrm{~L}$ pots and raised on half-strength nutrient solution for 7 days (Yoshida et al., 1976) (Table S1), after which it was replaced with full-strength nutrient solution. Nutrient solutions were renewed every 3 days and adjusted to a $\mathrm{pH}$ of 5.1 using $1 \mathrm{M} \mathrm{NaOH} / \mathrm{HCl}$ to simulate paddy soil conditions. Seedlings were allowed to grow for five weeks before treatment was initiated. For Cd treatment, seedlings were transferred to full-strength nutrient solution with $0.1,1.0$, or $10.0 \mu \mathrm{M} \mathrm{Cd}$ added to mimic low, moderate, and high-Cd stress conditions, respectively. $\mathrm{Cd}$ 
treatment was applied for three days. For the control condition, seedlings continued to receive full-strength nutrient solution without $\mathrm{Cd}$. The seedlings were grown in pairs in a hole with a diameter of $20 \mathrm{~mm}$ in a black foam plate. Each pot contained four pairs of seedlings for each cultivar, and three replicates were conducted for each treatment. The rice plants were allowed to grow eight weeks before root exudate collection. All pots were laid out in a phytotron with the following growth condition: $14 \mathrm{~h} / 26^{\circ} \mathrm{C}$ day $\left(250 \mu \mathrm{mol} \mathrm{m} \mathrm{s}^{-2}\right)$, $10 \mathrm{~h} / 20^{\circ} \mathrm{C}$ night, and $65 \%$ relative humidity.

\subsection{Root exudate collection and UPLC-ESI-Q-TOF-MS analysis}

After the Cd treatment, the rice root exudates were sampled according to Widodo et al. (2010). Briefly, three pairs of plants were collected from each pot and the roots were carefully rinsed with Milli-Q water. Each plant was then placed in a separate brown glass bottle containing $150 \mathrm{~mL}$ Milli-Q water and wrapped with aluminum foil to avoid degradation. After a $4 \mathrm{~h}$ collection period, a $40 \mathrm{~mL}$ aliquot was immediately lyophilized (FreeZone 12, LabConco, USA). An extracting solution of methanol/water $(200 \mu \mathrm{L}, 50: 50, \mathrm{v} / \mathrm{v})$ was added to the dried aliquots, sonicated $\left(20 \mathrm{~min}, 4^{\circ} \mathrm{C}\right)$, and centrifuged (15 min, $14000 \mathrm{~g}, 4^{\circ} \mathrm{C}$ ). A quality control sample was employed by mixing aliquots of all samples, which showed good reproducibility (Fig. S1). The total organic carbon (TOC) concentration and $\mathrm{pH}$ value of the root exudates were determined using a TOC analyzer (Vario TOC, Elementar, Germany) and a pH meter (FE28, Mettler Toledo), respectively.

Untargeted metabolic profiling was performed using an UPLC (ACQUITY, Waters, USA) coupled to a quadrupole time-of-flight mass spectrometry (Q-TOF-MS) instrument (SYNAPT G2-Si, Waters, USA). An electrospray ionization $\left(\mathrm{ESI}^{-}\right)$source was used for mass detection. Samples of root exudate metabolite extracts $(10 \mu \mathrm{L})$ were injected into the LC system and separated on a BEH C18 column $(2.1 \times 100 \mathrm{~mm}$, $1.7 \mu \mathrm{m}$, Waters). The detailed workflow for metabolic profiling and the operating conditions for the equipment are described in Text S1.

\subsection{Morphological analysis}

After collection of the exudates, the roots were imaged using a scanner (V750 Pro, Epson) set to high accuracy (800 dpi). The scanned images were analyzed using WinRHIZO software (version 2008a Pro, Regent Instruments Inc., Canada) to obtain root morphology parameters, including root tips (RT), total root length $(R L)$, crossings (RC), average diameter (RAD), forks (RF), surface area (RSA), and volume (RV). Next, the root tips/surface area ratio (NRT/SA) was calculated based on the measured root parameters. Lastly, the dry biomass of the plant was weighed after oven-drying at $70^{\circ} \mathrm{C}$ for $72 \mathrm{~h}$.
The viability of the RT was determined by staining the roots for 10 min with a fluorescent dye (Pan et al., 2001) comprising fluorescein diacetate $\left(12.5 \mathrm{~g} \mathrm{~mL}^{-1}\right)$ and propidium iodide $\left(5 \mathrm{~g} \mathrm{~mL}^{-1}\right)$. Fluorescence images of the RT were obtained using a fluorescence microscope (AF6000, Leica, Germany).

\subsection{Cd and nutrient element analysis}

Dried rice tissue was digested with $\mathrm{HNO}_{3}-\mathrm{H}_{2} \mathrm{O}_{2}$ solution (3:1, v:v) by a microwave digestion system (MARS 6, CEM, USA) at $180^{\circ} \mathrm{C}$ for $30 \mathrm{~min}$. Cd and nutrient elements were analyzed using ICP-OES (5300DV, PerkinEImer, USA) or ICP-MS (NexION, 350D PerkinEImer, USA). For ICP-MS analysis, $10 \mu \mathrm{g} \mathrm{L}^{-1}$ indium (In) was added to the samples as an internal standard and had a recovery rate of $95 \%-105 \%$. Certified reference samples GBW07602 (GSV-1) were employed and the recovery rates for metals were $88 \%-105 \%$.

\subsection{Statistical analysis}

Cd translocation from root to shoot was determined as the ratio of shoot $\mathrm{Cd}$ concentration to root $\mathrm{Cd}$ concentration. Oneway analysis of variance with Duncan's test was performed using SPSS 19.0 to determine the statistical significance of root morphological indexes and abundance of root exudate metabolites. The Pearson correlation analysis between root Cd concentration and TOC concentration in root exudates was performed by Origin (Pro 2021b, OriginLab Corporation, USA).

Based on the membership function, the rice root morphological indexes were integrated to calculate the Cd tolerance index of the rice roots (Khan et al., 2020). The Cd tolerance index (CTI) was calculated using the following formula:

$$
\begin{aligned}
& C T I_{i}=\frac{X-X_{\min }}{X_{\max }-X_{\min }} \\
& C T I=\frac{1}{n} \sum_{i=1}^{n} C T I_{i}
\end{aligned}
$$

where $C T I_{i}$ is the subordinate value, $X$ is the measured value of the root morphological indexes, and $X_{\max }$ and $X_{\min }$ are the maximum and minimum values of the root morphological indexes, respectively.

UPLC-ESI-Q-TOF-MS data processing was performed according to Petriacq et al. (2017). Prior to multivariate analyses, the raw UPLC-Q-TOF peaks were processed by normalization using the median, logarithm transformation, and Pareto scaling using MetaboAnalyst 4.0 (Xia et al., 2015). Supervised partial least square discriminant analysis (PLSDA) was employed to determine the separation of different treatments. A variable importance in projection (VIP)>1.0 and $P<0.05$ were selected as the criteria for separation, identified as significantly differential metabolites (SDMs). The SDMs were identified by referencing public databases (e.g., METLIN, MassBank). 


\section{Results}

\subsection{Effects of $\mathrm{Cd}$ on rice growth and root $\mathrm{Cd}$ accumulation}

The response of rice biomass to $\mathrm{Cd}$ was different between JY841 and TY816 plants (Fig. S2). Compared with the control condition, moderate $(1.0 \mu \mathrm{M}) \mathrm{Cd}$ treatment significantly inhibited rice growth in TY816 but not in JY841; by contrast, biomass increased in both cultivars in the low-Cd $(0.1 \mu \mathrm{M})$ treatment. However, when $\mathrm{Cd}$ stress was increased from moderate $(1.0 \mu \mathrm{M})$ to high $(10 \mu \mathrm{M})$, root and shoot biomass increased significantly $(23.1 \%$ and $20.6 \%$, respectively) in TY816, whereas JY841 showed no significant changes.

TY816 had significantly lower root $\mathrm{Cd}$ concentrations compared to JY841 under all Cd treatments (Fig. 1). To determine the effect of $\mathrm{Cd}$ on nutrient element uptake interference, the interactions of 10 elements in the roots of the two rice cultivars were analyzed. Intriguingly, the correlations of $\mathrm{Cd}$ with mineral elements in the roots differed between JY841 and TY816 (Fig. S3). For TY816, root Cd was positively correlated with $\mathrm{Ca}$ and $\mathrm{Zn}$ but negatively correlated with $\mathrm{Mg}$. By contrast, for JY841, root Cd was negatively correlated with $\mathrm{S}$ and positively correlated with $\mathrm{Ca}$.

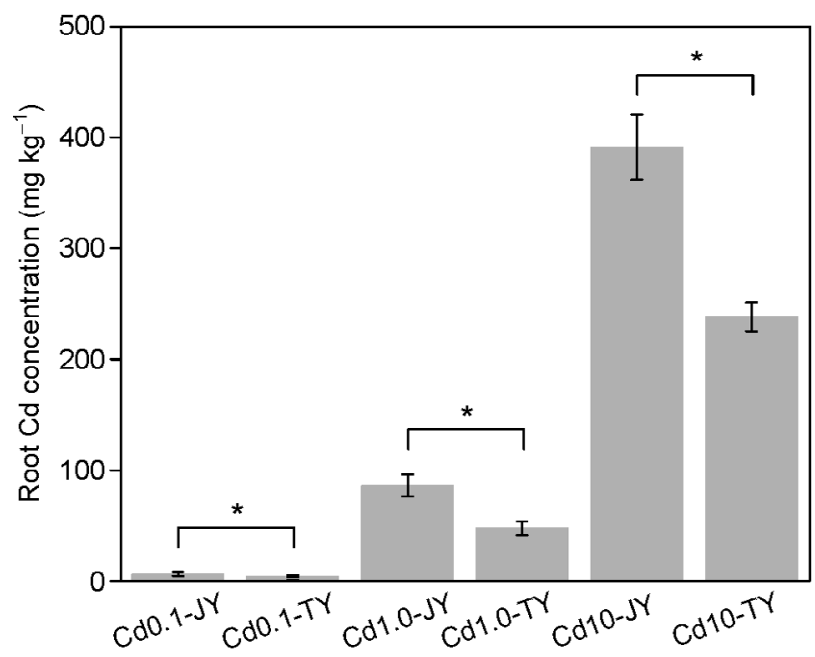

Fig. 1 Root $\mathrm{Cd}$ concentration in rice roots under different $\mathrm{Cd}$ treatments. CK-JY/TY, Cd0.1-JY/TY, Cd1.0-JY/TY, and Cd10-JY/ TY represent the control, $0.1 \mu \mathrm{M}, 1.0 \mu \mathrm{M}$, and $10 \mu \mathrm{M} \mathrm{Cd}$ treatment conditions in JY841 or TY816 cultivars, respectively. *, $P<0.05$.

\subsection{Phenomic response of the rice root systems}

To reveal the effects of $\mathrm{Cd}$ on the root systems of the two cultivars, a root morphology analysis and $\mathrm{Cd}$ tolerance evaluation were conducted (Table 1, Fig. 2). The Cd hormesis to the root morphological characteristics of TY816 was marginal, whereas low $\mathrm{Cd}(0.1 \mu \mathrm{M})$ significantly promoted root traits in JY841. Root length, surface area, root volume, root forks, and root crossings in TY816 were significantly promoted under $10 \mu \mathrm{M} \mathrm{Cd}$ compared with $1.0 \mu \mathrm{M} \mathrm{Cd}$

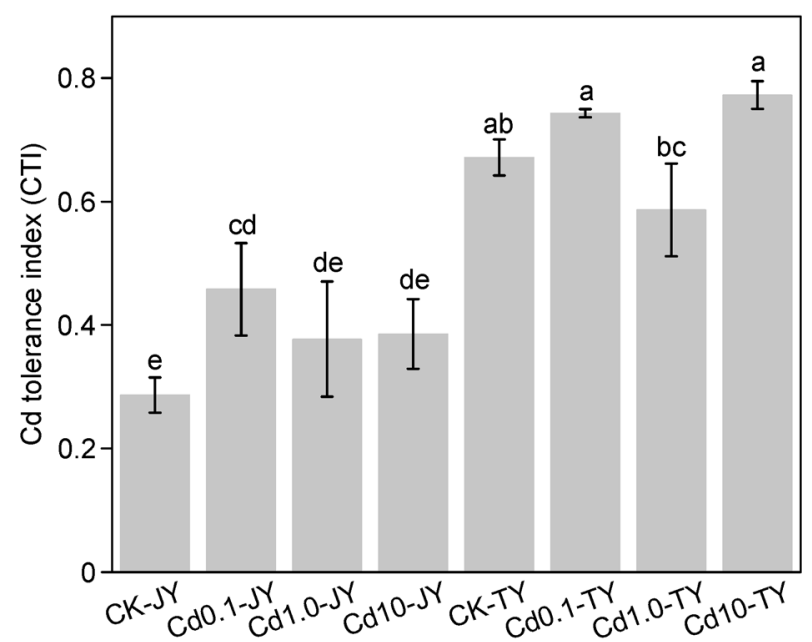

Fig. 2 Cd tolerance index of the roots of the two rice cultivars after three days of exposure to various levels of Cd stress.

treatment, while these measures were not significantly changed in JY841. The CTI of TY816 was significantly promoted under $10 \mu \mathrm{M}$ Cd compared with $1.0 \mu \mathrm{M} \mathrm{Cd}$ treatment whereas that of JY841 was unchanged, which is consistent with the biomass results.

To further dissect differences between the root systems of the two cultivars, the vitality of the root tips was determined. The root tip cells of both cultivars showed the highest viability in the control treatment, and some loss of viability was observed in the high-Cd treatment (Fig. 3). The root tip cells of TY816 showed no obvious damage, while most cells of JY841 lost viability.

\subsection{Response of root exudates to $\mathrm{Cd}$}

To reveal the effects of $\mathrm{Cd}$ on the root exudates of the two rice cultivars, the TOC concentration and $\mathrm{pH}$ value of the root exudates were determined. The TOC concentration in TY816 increased with increasing Cd stress, while only marginal changes were observed in JY841. There was a sharp reduction in the $\mathrm{pH}$ value of the root exudates of JY841, whereas the $\mathrm{pH}$ increased significantly in TY816 (Fig. S4). Correlation analysis showed no significant relationship between root $\mathrm{Cd}$ and TOC in JY841, while root $\mathrm{Cd}$ was positively correlated with TOC in the root exudate of TY816 $\left(R^{2}=0.82, P<0.0001\right)$ (Fig. S5). To further evaluate the different effects of $\mathrm{Cd}$ on the root exudates of the two rice cultivars, untargeted metabolic profiling analysis was employed. The overall variation in the metabolites of the root exudates between different treatments was visualized by principal component analysis (PCA) and PLS-DA, showing high correlation $\left(R^{2}=0.99\right)$ and predictability $\left(Q^{2}=0.94\right)$ (Fig. 4). Analysis revealed a clear separation in the first principal component for the composition of the root exudate metabolites, explaining $58.4 \%$ of the variation in the data. There were distinct differences in the polar metabolite 
Table 1 Root morphological characteristics in different treatments.

\begin{tabular}{|c|c|c|c|c|c|c|c|}
\hline Treatment & $\mathrm{RL}(\mathrm{cm})$ & $\mathrm{SA}\left(\mathrm{cm}^{2}\right)$ & $\mathrm{AD}(\mathrm{mm})$ & $\mathrm{RV}\left(\mathrm{cm}^{3}\right)$ & RF & $\mathrm{RC}$ & RT/SA \\
\hline CK-JY & $9314 \pm 460 d$ & $680 \pm 27 e$ & $0.234 \pm 0.021 \mathrm{c}$ & $3.984 \pm 0.522 c$ & $124613 \pm 8940 d$ & $22365 \pm 2327 b c$ & $139 \pm 16 a$ \\
\hline Cd0.1-JY & $10010 \pm 771 \mathrm{~cd}$ & $819 \pm 48 d$ & $0.261 \pm 0.029 b$ & $5.415 \pm 0.889 b$ & $151614 \pm 8497 a b c$ & $26423 \pm 4398 a b c$ & $107 \pm 19 b c$ \\
\hline Cd1.0-JY & $10191 \pm 425 b c$ & $696 \pm 41 \mathrm{e}$ & $0.217 \pm 0.011 \mathrm{c}$ & $3.795 \pm 0.372 c$ & $148578 \pm 14480 a b c$ & $30261 \pm 2584 a$ & $123 \pm 18 a b$ \\
\hline Cd10-JY & $9496 \pm 1275 b c$ & $702 \pm 75 e$ & $0.236 \pm 0.007 \mathrm{c}$ & $4.15 \pm 0.314 c$ & $132039 \pm 15664 \mathrm{~cd}$ & $25429 \pm 4350 a b c$ & $114 \pm 11 a b$ \\
\hline CK-TY & $11674 \pm 1152 a b$ & $1105 \pm 93 b c$ & $0.301 \pm 0.004 a$ & $8.347 \pm 0.568 a$ & $171645 \pm 14192 a$ & $26860 \pm 1866 a b$ & $84 \pm 5 d$ \\
\hline Cd0.1-TY & $10810 \pm 600 \mathrm{bc}$ & $1085 \pm 87 a$ & $0.318 \pm 0.01 a$ & $8.708 \pm 0.869 a$ & $156674 \pm 11367 a b$ & $23447 \pm 1043 b c$ & $85 \pm 6 d$ \\
\hline Cd1.0-TY & $9859 \pm 1045 b c$ & $934 \pm 154 c$ & $0.298 \pm 0.022 a$ & $7.138 \pm 1.604 b$ & $139260 \pm 15942 \mathrm{bcd}$ & $21505 \pm 1539 c$ & $95 \pm 14 \mathrm{~cd}$ \\
\hline Cd10-TY & $11336 \pm 740 a$ & $1058 \pm 99 \mathrm{ab}$ & $0.296 \pm 0.01 a$ & $7.907 \pm 1.027 a$ & $162820 \pm 12017 a$ & $26035 \pm 1631 \mathrm{ab}$ & $89 \pm 5 \mathrm{~cd}$ \\
\hline
\end{tabular}

$\mathrm{RL}, \mathrm{RSA}, \mathrm{RV}, \mathrm{RT}, \mathrm{AD}, \mathrm{RF}$ and RC presents total root length, root surface area, root volume average, diameter, root forks, and root crossings, respectively. Means within rows followed by different letters are significantly different according to Duncan 's multiple test $(P \leqslant 0.05)$.

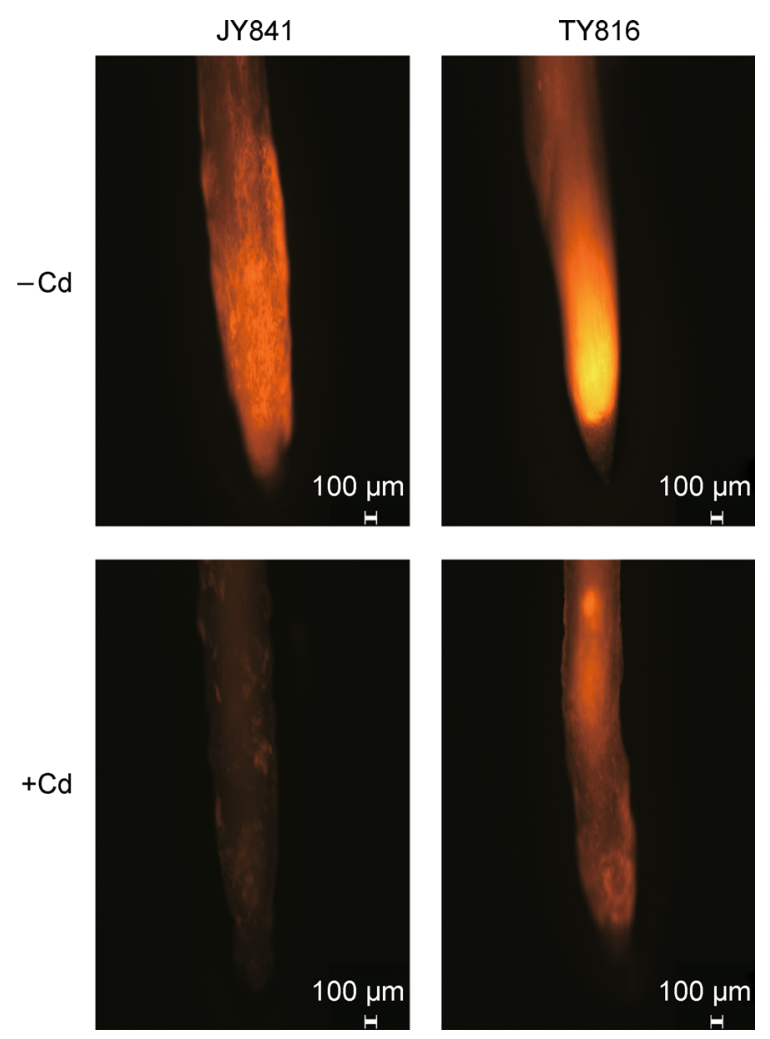

Fig. 3 Visualization of the viability of root tips in rice seedlings exposed to $\mathrm{Cd}(10 \mu \mathrm{M})$. The intense fluorescence indicates high activity of the cells.

composition of the root exudates between the two rice cultivars under control or $10 \mu \mathrm{M} \mathrm{Cd}$ treatment conditions in our cultivation system.

To further distinguish the Cd-induced root exudates, PLSDA analysis of the metabolic data was carried out separately for the two rice cultivars under $\mathrm{Cd}$ stress. As a result, cultivarspecific SDMs and SDMs shared by the two cultivars were identified (Fig. S6). Under Cd stress, the majority of affected compounds were common to both rice varieties (18 SDMs,
$75 \%$ ), while several were cultivar-specific (6 SDMs, $25 \%$ ). For the shared Cd-induced SDMs, there were 12 shared downregulated SDMs and 6 shared upregulated SDMs. For the cultivar-specific Cd-induced SDMs, 5 SDMs (2-hydroxynonadecanoic acid, $\mathrm{PC}(18: 3(6 Z, 9 Z, 12 Z) / 0: 0), \mathrm{PS}(22: 2$ $(13 Z, 16 Z) / 21: 0), P A(O-20: 0 / 16: 0)$, and oryzalexin $B)$ were downregulated in JY841, but upregulated in TY816 (Fig. 5). Only one SDM (Orobanchoside) was upregulated by $\mathrm{Cd}$ in JY841, whereas it was downregulated by Cd in TY816.

\section{Discussion}

4.1 Contrasting root phenotypes regulate $\mathrm{Cd}$ uptake in two rice cultivars

Root phenotypes are the result of evolutionary selection for efficient acquisition of endogenous soil resources and resistance to environmental stresses (Neumann and Martinoia, 2002; Wang et al., 2015; Lynch, 2019). Cd uptake has been reported to affect root phenotypes and vice versa (Redjala et al., 2011). Exposure to $\mathrm{Cd}$ can modify root architecture by affecting a range of processes, including nutrient uptake, hormonal signal transport, and gene expression, consequently affecting lateral root growth (Boursiac et al., 2013; Wen et al., 2019). Correspondingly, under Cd stress, plants improve their metal tolerance ability by reconstructing their root architecture (Lu et al., 2013). We found that rice with a lower root tips/surface area ratio (RT/SA) had lower $\mathrm{Cd}$ uptake, suggesting that RT/RSA rather than RSA alone is pivotal for Cd absorption and migration (Huang et al., 2019). RT/RSA or apical surface area may affect the absorption of $\mathrm{Cd}$, thus determining Cd concentration in roots (Redjala et al., 2011). The root tip is not only the target toxic action site for $\mathrm{Cd}$, it is also the most active position for $\mathrm{Cd}^{2+}$ influx into roots (Tao et al., 2016). We also observed that the TY816 cultivar had a greater RSA, average diameter, and volume compared to the JY841 cultivar. Liang et al. (2017) found that the RSA and volume of spring wheat under $\mathrm{Cd}$ treatment were positively 

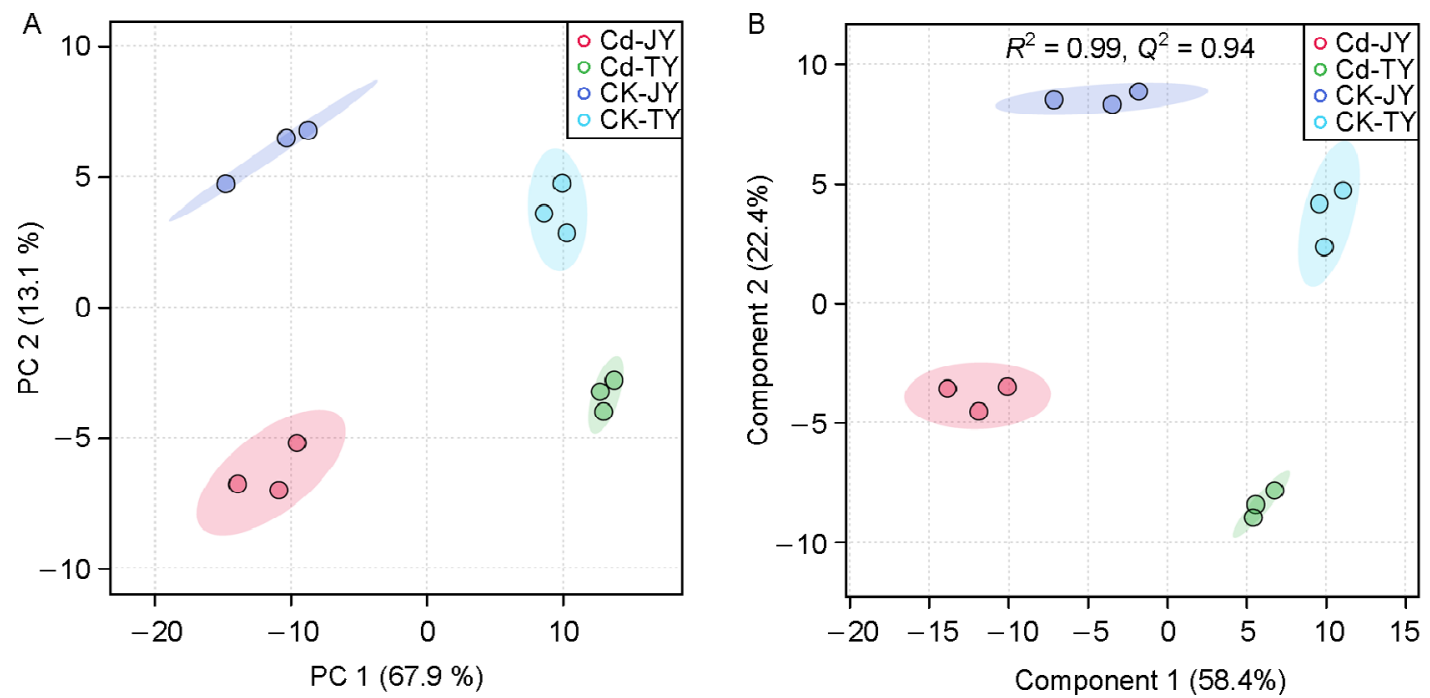

Fig. 4 Variation in root exudate metabolites under different treatment conditions. (A) Principal component analysis (PCA) and (B) supervised partial least square discriminant analysis (PLS-DA). CK-JY/TY and Cd-JY/TY represent the control and $10 \mu \mathrm{M}$ Cd treatment for JY841/TY816, respectively.

correlated with grain $\mathrm{Cd}$ concentration, from which they concluded that $\mathrm{Cd}$ retention in roots did not contribute to differences in $\mathrm{Cd}$ grain concentration. However, Harris and Taylor (2013) showed that root system traits were negatively correlated with $\mathrm{Cd}$ concentration in the grains and shoots of durum wheat. Compared to JY841, TY816 is privileged with a stronger root system characterized by a greater surface area, average root diameter, and volume, but a lower apical surface area, under high-Cd $(10 \mu \mathrm{M})$ stress. The low-Cd cultivar TY816 had a lower apical surface area and lower maximum uptake rate of $\mathrm{Cd}$ relative to the high-Cd JY841, which resulted in lower Cd accumulation in its root (Huang et al., 2019). Plants with well-developed roots possess a wider attachment area, leading to greater oxygen release, metal adsorption, and/or coprecipitation (Rajkumar et al., 2010; Wang et al., 2015). Furthermore, a stronger root system can release various hormones and induce fluctuations in the rhizosphere and root architecture reconstruction, thereby influencing Cd uptake by roots (Li et al., 2009).

\subsection{Contrasting regulation of root exudates of two rice cultivars under $\mathrm{Cd}$ stress}

Root exudates have proved to play an important role in $\mathrm{Cd}$ detoxification in plants. The present study found contrasting regulation of root exudates between the two rice varieties differing in their $\mathrm{Cd}$ accumulation ability. Significant rhizosphere acidification was observed in the high-Cd accumulation cultivar JY841, while alkalization was observed in the lowCd accumulation cultivar TY816 (Fig. S4). Sun et al. (2019) found that rhizosphere acidification promotes $\mathrm{Cd}$ uptake via the Cd hyperaccumulator Sedum plumbizincicola. Therefore, root exudate-induced changes in rhizosphere $\mathrm{pH}$ may contribute to differences in $\mathrm{Cd}$ uptake by different rice varieties. In addition, a positive correlation of root $\mathrm{Cd}$ concentration with TOC of root exudates was observed in TY816, indicating that this cultivar may exhibit a detoxification or defensive reaction in response to Cd stress (Zhao et al., 2016). The root exudates of fatty acids and lipids including, 2hydroxy-nonadecanoic acid, PC(18:3(6Z,9Z,12Z)/0:0), PS $(22: 2(13 Z, 16 Z) / 21: 0)$, and $P A(O-20: 0 / 16: 0)$, were all downregulated by $\mathrm{Cd}$ in JY841 but upregulated in TY816. When roots are stressed by heavy metals, the cellular responses that occur include fatty acid release from membranes and variation in lipid amounts (Upchurch, 2008). The concentrations of fatty acids and lipids in the root exudate of TY816 were significantly upregulated, indicating active defense of root membranes to $\mathrm{Cd}$ stress. Cd-induced disturbances in membrane lipid turnover and lipid composition might promote the metabolism of fatty acids (Nouairi et al., 2006; Debiane et al., 2012). The oxidative stress caused by $\mathrm{Cd}$ is known to accelerate the desaturation of membrane lipids and increase the level of unsaturated fatty acids, which may help resist membrane damage induced by Cd (Zemanová et al., 2015). Orobanchoside was upregulated by $\mathrm{Cd}$ in JY841, whereas it was downregulated in TY816. This difference could be due to the depletion of sugar, which acts as a reactive oxygen species scavenger in root tissue (Keunen et al., 2013). This study also found that the secondary metabolite oryzalexin B was downregulated by Cd in JY841 but upregulated in TY816. Growing evidence suggests that flavonoid compounds play an active role in metal stress response and anti-lipid peroxidation via free radical scavenging and metal chelation (Li et al., 2015). 

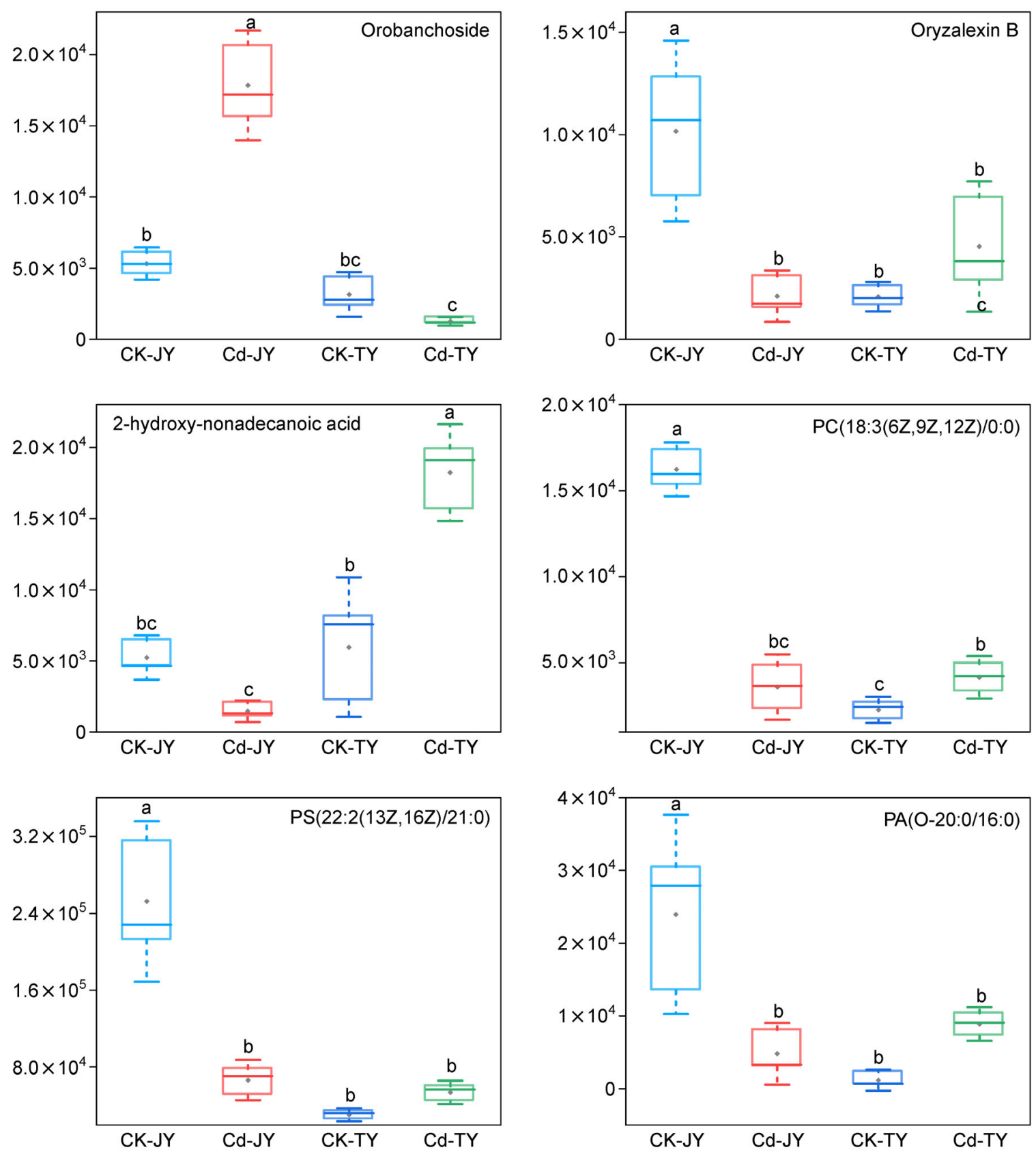

Fig. 5 Box plots of the relative abundance of root exudate metabolites in the two rice cultivars under $10 \mu \mathrm{M} \mathrm{Cd}$ treatment $(n=3)$. The mean concentration of each group is indicated by a diamond.

4.3 Root architecture and exudates mediate the contrasting responses of two rice cultivars to $\mathrm{Cd}$

Roots present the first barrier to $\mathrm{Cd}$, which accumulates in roots and is translocated to shoots (Uraguchi and Fujiwara, 2013). Since most of the accumulated $\mathrm{Cd}$ is reserved in the rice root system, variation in the sink has a great influence on Cd levels in shoots and grains (Harris and Taylor, 2013). In this study, we found that some root traits of TY816 were significantly enhanced by $10 \mu \mathrm{M} C d$ and that lower $\mathrm{Cd}$ uptake was observed, indicating that root barriers may contribute to lower levels of $\mathrm{Cd}$ in TY816. Plants shape their roots and secrete a wide variety of metabolites into the rhizosphere to cope with heavy metal stress. Therefore, contrasting $\mathrm{Cd}$ accumulation abilities among different rice cultivars may be due to differences in the reprogramming of root architecture and root exudates (Xin et al., 2017). It has been reported that improvement in root traits can accelerate the uptake of mineral elements and the transpiration rate (Ding et al., 2014). Although RT lack a Casparian strip, making them the most active region for $\mathrm{Cd}^{2+}$ influx into plant roots (Tao et al., 2016), most root exudates are localized at the root tip (Canarini et al., 
2019). Thus, the difference in $\mathrm{Cd}$ accumulation between JY841 and TY816 may be determined not only by the root architecture but also by the root exudates. Although there have been many studies on root architecture and root exudates under heavy metal stress (Redjala et al., 2011; Zhao et al., 2018; Huang et al., 2019), these issues have rarely been linked to predict rhizosphere resistance strategies. Our study found contrasting responses of root traits and root exudate metabolites to $\mathrm{Cd}$ stress between different rice cultivars. This finding suggests that rice roots can increase their tolerance to $\mathrm{Cd}$ stress by simultaneously employing multiple strategies involving metabolomics and phenotypes. Despite the fact that the rhizosphere is an active area with high dynamics, the extent and shape of root growth, element fluxes, and exudates are quasi-stationary due to the opposite formation process (Kuzyakov and Razavi, 2019). Cd exhibits a rhizosphere extent $<2 \mathrm{~mm}$, whereas root exudates have a rhizosphere extent of 2-4 mm (Duan et al., 2018). Thus, it appears that there is no intersection between the extent of $\mathrm{Cd}$ and root exudates in the rhizosphere. However, root exudation metabolites are driven by diffusion. RT can sense variation in exudation concentration and change the architecture of the root system through signal transduction (Canarini et al., 2019). Therefore, changes in root morphology induced by $\mathrm{Cd}$ stress can in turn affect root $\mathrm{Cd}$ uptake by influencing the mobilization of $\mathrm{Cd}$ in the rhizosphere (Li et al., 2009).

\section{Conclusions}

Different rhizospheric resistance strategies to cope with $\mathrm{Cd}$ stress were identified between two rice varieties differing in their $\mathrm{Cd}$ accumulation ability. The combination of metabolomic and phenomic responses revealed that the roots of the low-Cd rice cultivar (TY816) employ multiple strategies to enhance their tolerance of $\mathrm{Cd}$-induced oxidative stress, including alteration of root system architecture, adjustment of membrane lipid turnover, and release of secondary metabolites to prevent lipid peroxidation by scavenging free radicals and metal chelation. Compared with TY816, the high-Cd cultivar (JY841) suffered serious root membrane damage and showed upregulation of phenylethanoid glycosides to cope with Cd-induced oxidative stress. Taken together, these findings have further increased our understanding of root architecture and exudate-mediated $\mathrm{Cd}$ resistance in rice. These findings will assist not only in supplementing external $\mathrm{Cd}$ resistance mechanisms in the rhizosphere, but also in manipulating rhizosphere architecture and metabolites to mitigate $\mathrm{Cd}$ contamination in rice.

\section{Acknowledgments}

This work was supported by the National Key R\&D Program of China (2018YFD0800700), the National Natural Science Foundation of China (41877121, 41920104003), and the 111 Project (B18060).

\section{Electronic supplementary material}

Supplementary material is available in the online version of this article at https://doi.org/10.1007/s42832-021-0088-0 and is accessible for authorized users.

\section{References}

Boursiac, Y., Léran, S., Corratgé-Faillie, C., Gojon, A., Krouk, G., Lacombe, B., 2013. ABA transport and transporters. Trends in Plant Science 18, 325-333.

Canarini, A., Kaiser, C., Merchant, A., Richter, A., Wanek, W., 2019. Root exudation of primary metabolites: mechanisms and their roles in plant responses to environmental stimuli. Frontiers in Plant Science 10, 1-19.

Chen, Y.T., Wang, Y., Yeh, K.C., 2017. Role of root exudates in metal acquisition and tolerance. Current Opinion in Plant Biology 39, 6672.

Chi, Y., Li, F., Tam, N.F.y., Liu, C., Ouyang, Y., Qi, X., Li, W.C., Ye, Z., 2018. Variations in grain cadmium and arsenic concentrations and screening for stable low-accumulating rice cultivars from multienvironment trials. Science of the Total Environment 643, 13141324.

Debiane, D., Calonne, M., Fontaine, J., Laruelle, F., GrandmouginFerjani, A., Lounès-Hadj Sahraoui, A., 2012. Benzo[a]pyrene induced lipid changes in the monoxenic arbuscular mycorrhizal chicory roots. Journal of Hazardous Materials 209-210, 18-26.

Ding, Y., Feng, R., Wang, R., Guo, J., Zheng, X., 2014. A dual effect of Se on Cd toxicity: evidence from plant growth, root morphology and responses of the antioxidative systems of paddy rice. Plant and Soil 375, 289-301.

Duan, C., Fang, L., Yang, C., Chen, W., Cui, Y., Li, S., 2018. Reveal the response of enzyme activities to heavy metals through in situ zymography. Ecotoxicology and Environmental Safety 156, 106115.

Fu, H., Yu, H., Li, T., Zhang, X., 2018. Influence of cadmium stress on root exudates of high cadmium accumulating rice line (Oryza sativa L.). Ecotoxicology and Environmental Safety 150, 168-175.

Harris, N.S., Taylor, G.J., 2013. Cadmium uptake and partitioning in durum wheat during grain filling. BMC Plant Biology 13, 103.

Hu, Y., Cheng, H., Tao, S., 2016. The challenges and solutions for cadmium-contaminated rice in China: a critical review. Environment International 92-93, 515-532.

Huang, B., Xin, J., Dai, H., Liu, A., Zhou, W., Yi, Y., Liao, K., 2015. Root morphological responses of three hot pepper cultivars to $\mathrm{Cd}$ exposure and their correlations with $\mathrm{Cd}$ accumulation. Environmental Science and Pollution Research International 22, 11511159.

Huang, L., Li, W.C., Tam, N.F.Y., Ye, Z., 2019. Effects of root morphology and anatomy on cadmium uptake and translocation in rice (Oryza sativa L.). Journal of Environmental Sciences (China) 75, 296-306.

Keunen, E., Peshev, D., Vangronsveld, J., Van Den Ende, W., Cuypers, A., 2013. Plant sugars are crucial players in the oxidative 
challenge during abiotic stress: extending the traditional concept. Plant, Cell \& Environment 36, 1242-1255.

Khan, J., Yang, Y., Fu, Q., Shao, W., Wang, J., Shen, L., Huai, Y., Malangisha, G. K., Ali, A., Mahmoud, A., 2020. Screening of watermelon varieties for lead tolerance at the seedling stage HortScience 55, 858-869.

Kuzyakov, Y., Razavi, B.S., 2019. Rhizosphere size and shape: Temporal dynamics and spatial stationarity. Soil Biology \& Biochemistry 135, 343-360.

Li, H., Luo, N., Li, Y.W., Cai, Q.Y., Li, H.Y., Mo, C.H., Wong, M.H., 2017. Cadmium in rice: Transport mechanisms, influencing factors, and minimizing measures. Environmental Pollution 224, 622-630.

Li, J., Lu, H., Liu, J., Hong, H., Yan, C., 2015. The influence of flavonoid amendment on the absorption of cadmium in Avicennia marina roots. Ecotoxicology and Environmental Safety 120, 1-6.

Li, T., Yang, X., Lu, L., Islam, E., He, Z., 2009. Effects of zinc and cadmium interactions on root morphology and metal translocation in a hyperaccumulating species under hydroponic conditions. Journal of Hazardous Materials 169, 734-741.

Liang, X., Strawn, D.G., Chen, J., Marshall, J., 2017. Variation in cadmium accumulation in spring wheat cultivars: uptake and redistribution to grain. Plant and Soil 421, 219-231.

Lin, Q., Chen, Y.X., Chen, H.M., Yu, Y.L., Luo, Y.M., Wong, M.H., 2003. Chemical behavior of $\mathrm{Cd}$ in rice rhizosphere. Chemosphere 50 , 755-761.

Liu, J., Qian, M., Cai, G., Zhu, Q., Wong, M.H., 2007. Variations between rice cultivars in root secretion of organic acids and the relationship with plant cadmium uptake. Environmental Geochemistry and Health 29, 189-195.

Lu, Z., Zhang, Z., Su, Y., Liu, C., Shi, G., 2013. Cultivar variation in morphological response of peanut roots to cadmium stress and its relation to cadmium accumulation. Ecotoxicology and Environmental Safety $91,147-155$.

Lynch, J.P., 2019. Root phenotypes for improved nutrient capture: an underexploited opportunity for global agriculture. New Phytologist 223, 548-564.

Neumann, G., Martinoia, E., 2002. Cluster roots-an underground adaptation for survival in extreme environments. Trends in Plant Science 7, 162-167.

Nouairi, I., Ghnaya, T., Ben Youssef, N., Zarrouk, M., Habib Ghorbel, M., 2006. Changes in content and fatty acid profiles of total lipids of two halophytes: Sesuvium portulacastrum and Mesembryanthemum crystallinum under cadmium stress. Journal of Plant Physiology 163, 1198-1202.

Pan, J., Zhu, M., Chen, H., 2001. Aluminum-induced cell death in roottip cells of barley. Environmental and Experimental Botany 46, 7179.

Pétriacq, P., Williams, A., Cotton, A., McFarlane, A.E., Rolfe, S.A., Ton, J., 2017. Metabolite profiling of non-sterile rhizosphere soil. Plant Journal 92, 147-162.

Rajkumar, M., Ae, N., Prasad, M.N.V., Freitas, H., 2010. Potential of siderophore-producing bacteria for improving heavy metal phytoextraction. Trends in Biotechnology 28, 142-149.

Redjala, T., Zelko, I., Sterckeman, T., Leguv, V., Lux, A., 2011. Relationship between root structure and root cadmium uptake in maize. Environmental and Experimental Botany 71, 241-248.

Sun, X., Li, Z., Wu, L., Christie, P., Luo, Y., Fornara, D.A., 2019. Rootinduced soil acidification and cadmium mobilization in the rhizosphere of Sedum plumbizincicola: evidence from a high-resolution imaging study. Plant and Soil 436, 267-282.

Tao, Q., Hou, D., Yang, X., Li, T., 2016. Oxalate secretion from the root apex of Sedum alfredii contributes to hyperaccumulation of $\mathrm{Cd}$. Plant and Soil 398, 139-152.

Tao, Q., Zhao, J., Li, J., Liu, Y., Luo, J., Yuan, S., Li, B., Li, Q., Xu, Q., Yu, X., Huang, H., Li, T., Wang, C., 2020. Unique root exudate tartaric acid enhanced cadmium mobilization and uptake in Cdhyperaccumulator Sedum alfredii. Journal of Hazardous Materials 383, 121177.

Ueno, D., Yamaji, N., Kono, I., Huang, C.F., Ando, T., Yano, M., Ma, J. F., 2010. Gene limiting cadmium accumulation in rice. Proceedings of the National Academy of Sciences of the United States of America 107, 16500-16505.

Upchurch, R.G., 2008. Fatty acid unsaturation, mobilization, and regulation in the response of plants to stress. Biotechnology Letters 30, 967-977.

Uraguchi, S., Fujiwara, T., 2013. Rice breaks ground for cadmium-free cereals. Current Opinion in Plant Biology 16, 328-334.

Walker, T.S., Bais, H.P., Grotewold, E., Vivanco, J.M., 2003. Root exudation and rhizosphere biology. Plant Physiology 132, 44-51.

Wang, X., Tam, N.F.Y., He, H., Ye, Z., 2015. The role of root anatomy, organic acids and iron plaque on mercury accumulation in rice. Plant and Soil 394, 301-313.

Wen, Z., Li, H., Shen, Q., Tang, X., Xiong, C., Li, H., Pang, J., Ryan, M. H., Lambers, H., Shen, J., 2019. Tradeoffs among root morphology, exudation and mycorrhizal symbioses for phosphorusacquisition strategies of 16 crop species. New Phytologist 223, 882-895.

Widodo., Broadley, M.R., Rose, T., Frei, M., Pariasca-Tanaka, J., Yoshihashi, T., Thomson, M., Hammond, J.P., Aprile, A., Close, T. J., Ismail, A.M., Wissuwa, M., 2010. Response to zinc deficiency of two rice lines with contrasting tolerance is determined by root growth maintenance and organic acid exudation rates, and not by zinc-transporter activity. New Phytologist 186, 400-414.

Xia, J., Sinelnikov, I.V., Han, B., Wishart, D.S., 2015. MetaboAnalyst 3.0-making metabolomics more meaningful. Nucleic Acids Research 43, W251-7.

Xin, J., Huang, B., Dai, H., Mu, Y., 2017. Characterization of root morphology and root-derived low molecular weight organic acids in two sweet potato cultivars exposed to cadmium. Archives of Agronomy and Soil Science 63, 723-734.

Yoshida, S., Forno, D., Cock, J., Gomez, K., 1976. Laboratory manual for physiological studies of rice. Manila, Philippines.

Zemanová, V., Pavlík, M., Kyjaková, P., Pavlíková, D., 2015. Fatty acid profiles of ecotypes of hyperaccumulator Noccaea caerulescens growing under cadmium stress. Journal of Plant Physiology 180, 27-34.

Zhang, H., Lu, L., Zhao, X., Zhao, S., Gu, X., Du, W., Wei, H., Ji, R., Zhao, L., 2019. Metabolomics reveals the "invisible" responses of spinach plants exposed to $\mathrm{CeO}_{2}$ nanoparticles. Environmental Science \& Technology 53, 6007-6017.

Zhao, F.J., Ma, Y., Zhu, Y.G., Tang, Z., McGrath, S.P., 2015. Soil 
contamination in China: current status and mitigation strategies. Environmental Science \& Technology 49, 750-759.

Zhao, J.Y., Ye, Z.H., Zhong, H., 2018. Rice root exudates affect microbial methylmercury production in paddy soils. Environmental Pollution 242, 1921-1929.

Zhao, L., Huang, Y., Hu, J., Zhou, H., Adeleye, A.S., Keller, A.A., 2016. ${ }^{1} \mathrm{H}$ NMR and GC-MS based metabolomics reveal defense and detoxification mechanism of cucumber plant under nano-Cu stress. Environmental Science \& Technology 50, 2000-2010.

Zhu, X.F., Zheng, C., Hu, Y.T., Jiang, T., Liu, Y., Dong, N.Y., Yang, J.L., Zheng, S.J., 2011. Cadmium-induced oxalate secretion from root apex is associated with cadmium exclusion and resistance in Lycopersicon esulentum. Plant, Cell \& Environment 34, 10551064. 